\title{
Efficient optomechanical cooling in one-dimensional interferometers
}

\author{
P. Domokos ${ }^{a}$, A. Xuereb ${ }^{b}$, P. Horak ${ }^{c}$, and T. Freegarde ${ }^{b}$ \\ ${ }^{a}$ Research Institute of Solid State Physics and Optics, H-1525 Budapest P.O. Box 49, Hungary; \\ ${ }^{b}$ School of Physics and Astronomy, University of Southampton, Southampton SO17 1BJ, UK; \\ ${ }^{c}$ Optoelectronics Research Centre, University of Southampton, Southampton SO17 1BJ, UK
}

\begin{abstract}
We present a scattering model which enables us to describe the mechanical force, including the velocity dependent component, exerted by light on polarizable massive objects in a general one-dimensional optical system. We show that the light field in an interferometer can be very sensitive to the velocity of a moving scatterer. We construct a new efficient cooling scheme, 'external cavity cooling', in which the scatterer, that can be an atom or a moving micromirror, is spatially separated from the cavity.
\end{abstract}

Keywords: Opto-mechanical systems, cavity cooling, scattering model, mechanical effect of light, laser cooling.

\section{INTRODUCTION}

Opto-mechanics ${ }^{1}$ is a rapidly growing field covering numerous physical realizations in which the gross motion of scatterers is manipulated by making use of the mechanical effects of light. The ponderomotive force exhibits a velocity-dependent character which stems from the retardation in the electromagnetic field inherent in such systems. ${ }^{2}$ For an appropriate choice of parameters, velocity-dependent terms in the force may lead to a viscous damping of motion. Opto-mechanical cooling of this type is a very general mechanism, and might turn out to be a necessary intermediate step to observe quantum effects on the mesoscopic scale, e.g., with micro-mechanical mirrors. ${ }^{3}$

However, pure Doppler frequency shifting results in a velocity dependent force proportional to $v / c$, which is negligibly small at room temperature and below. The well-known and very successful laser cooling of atoms, for example, produces a significant cooling effect because it relies on a resonant enhancement with the $Q$-factor $\omega / \gamma$ characteristic of an atomic transition ( $\omega$ is the frequency of the radiation, $\gamma$ is the linewidth of the transition). In non-resonant scatterers, the sensitivity of the radiation force to the velocity can be enhanced similarly, by coupling the moving object to a resonant optical element. This is the case, for example, in cavity cooling of atoms $^{4,5}$ where the motional energy of the atom is dissipated through a coupled, lossy cavity mode having a relatively large $Q$-factor $\left(Q=\omega_{C} / \kappa ; \omega_{C}\right.$ is the mode resonance frequency and $\kappa$ is its linewidth). This is also the case in several recent opto-mechanical cooling experiments: ${ }^{3,6-9}$ the thermal vibration of one of the micromirrors making up a Fabry-Pérot-type resonator can be quenched through the radiation pressure of the light field enclosed in the resonator.

The models used to describe the way light interacts with microscopic objects such as atoms and macroscopic objects such as mirrors have been completely different. In the first case, theories are based on the assumption that atoms are very weak scatterers in free space, negligibly perturbing the impinging bright laser beams. In the other case, the influence of the moving massive component on the radiation field is so strong that it is considered a (moving) boundary condition defining a single or a few modes of the field participating in the opto-mechanical coupling. ${ }^{10,11}$

We developed a new scattering theory for optomechanical systems that is able to describe the behavior of both scenarios using a single, unified framework. The approach is a generalization of the well-known transfer matrix method, ${ }^{12-14}$ which relates forward- and backward-going waves on both sides of an optical element, i.e.,

Send correspondence to P.D.: E-mail: domokos@szfki.hu 
the local relation between light field amplitudes at the two sides of a scatterer. The generalization consists in the determination of the transfer matrix of moving scatterers. In this scattering model, atoms and mirrors differ only in the value of the parameter describing their scattering strength. However, all the expressions derived from the scattering model encompass both limiting cases of atoms and moving mirrors and arbitrary combinations of them. The main virtue of this approach is that one can envisage and describe sophisticated interferometric setups in which the strength of these viscous forces might be greatly enhanced. Indeed we show that the fields in multiple-pass interferometers, such as the Fabry-Pérot cavity, exhibit great sensitivity not only to the presence but also to the motion of any scattering object within the optical path.

In Section 2 of this paper, we consider the most general case of an interferometer comprising an arbitrary configuration of generic 'beam splitters' and calculate the velocity-dependent radiation field and the light force exerted on a moving scatterer. Then, in Section 3, we will consider the system composed of two mirrors; one of them is fixed in space, whilst the other one is mobile. ${ }^{15}$ This is the generic scheme for radiation-pressure cooling of moving mirrors. ${ }^{16-18}$ At the same time, in the limit of low reflection the moving mirror can equally well represent a single atomic dipole interacting with its mirror image in front of a highly reflecting surface. ${ }^{19-21}$ The scattering model description of this example illustrates the use of the general expressions. In Section 4, we find that a simple configuration, in which the scatterer interacts with an optical resonator from which it is spatially separated, can enhance the optomechanical friction by several orders of magnitude with respect to the mirror-mediated cooling scheme presented in the previous section. ${ }^{22}$ This simple geometry illustrates the role of interference in damping the motion of a scatterer. A scatterer in front of a two-mirror resonator interacts with both an incoming laser beam and its reflection from the resonator. This reflected field can be thought of as a superposition of waves formed by multiple reflections between the mirrors of the cavity. With this scheme, which we label 'external cavity cooling', one can benefit from the large finesse of the cavity even if the moving object has a low reflectivity and construct a practically useful optical cooling method.

\section{ONE-DIMENSIONAL SCATTERING MODEL}

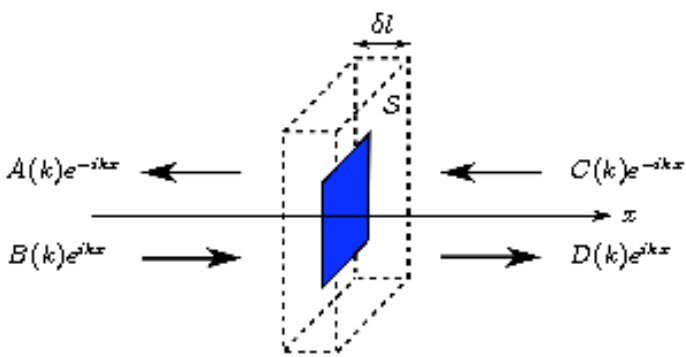

Figure 1. Schematic representation of the scatterer (beam splitter, BS) in one dimension. The scatterer combines the incoming waves, $B, C$ into the outgoing ones, $A, D$. Because the BS is moving, plane waves with different wave vectors $k$ are mixed. The model deals with the set of amplitudes $A(k), B(k)$, etc. The BS is surrounded by a fictitious volume with surface $S$ and width $\delta l$, which is used to calculate the force acting on the BS from the Maxwell stress tensor.

Consider a point-like scatterer (or beamsplitter), BS, moving along the ' $x$ ' axis on the trajectory $x_{\mathrm{BS}}(t)$. Outside the scatterer, the electric field $\boldsymbol{E}$ can be expressed in terms of a discrete sum of left- and right-propagating plane wave modes with different wave numbers, $k$, and hence different frequencies, $\omega=k c$ :

$$
\boldsymbol{E}= \begin{cases}\sum_{k}\left[A(k) e^{-i k x-i \omega t}+B(k) e^{i k x-i \omega t}\right]+\text { c.c. } & \text { if } x<x_{\mathrm{BS}}(t) \\ \sum_{k}\left[C(k) e^{-i k x-i \omega t}+D(k) e^{i k x-i \omega t}\right]+\text { c.c. } & \text { if } x>x_{\mathrm{BS}}(t),\end{cases}
$$

where $A(k)$ and $B(k)$ are the mode amplitudes on the left, while $C(k)$ and $D(k)$ are the amplitudes on the right side of BS. In accordance, the magnetic field is

$$
c \boldsymbol{B}= \begin{cases}\sum_{k}\left[-A(k) e^{-i k x-i \omega t}+B(k) e^{i k x-i \omega t}\right]+\text { c.c. } & \text { if } x<x_{\mathrm{BS}}(t) \\ \sum_{k}\left[-C(k) e^{-i k x-i \omega t}+D(k) e^{i k x-i \omega t}\right]+\text { c.c. } & \text { if } x>x_{\mathrm{BS}}(t) .\end{cases}
$$


As depicted schematically in Fig. 1, the scatterer mixes these waves. Our first goal is the derivation of the transfer matrix $M$ connecting the field amplitudes on the right to those on the left side of a beamsplitter.

\subsection{The scatterer}

The transfer matrix is known ${ }^{12}$ for an immobile scatterer, $v=0$,

$$
\left(\begin{array}{l}
C \\
D
\end{array}\right)=M_{0}\left(\begin{array}{l}
A \\
B
\end{array}\right), \text { with } M_{0}=\left[\begin{array}{cc}
1-i \zeta & -i \zeta \\
i \zeta & 1+i \zeta
\end{array}\right]
$$

which expresses that the scatterer can be described by a single parameter, the polarizability $\zeta$. It is connected to the customarily used parameters of a BS, to the reflectivity and transmissivity, as

$$
\mathfrak{r}=\frac{i \zeta}{1-i \zeta}, \quad \mathfrak{t}=\frac{1}{1-i \zeta} .
$$

Moreover, the use of polarizability makes the description very general: it can be connected to a single atom with linear polarizability $\alpha$ by $\zeta=\frac{\pi \alpha}{\epsilon_{0} \lambda S}$ where $S$ is the effective beam cross section. For a two-level, unsaturated atom with transition frequency $\omega_{\mathrm{A}}$ and linewidth $\Gamma$ (HWHM), for example,

$$
\zeta=\frac{\sigma_{\mathrm{A}}}{2 S} \frac{\Gamma}{\omega_{\mathrm{A}}-\omega-i \Gamma}
$$

where $\sigma_{\mathrm{A}}=\frac{3 \lambda^{2}}{2 \pi}$ is the resonant radiative cross section of an atom. The two-level atom is an example for which the polarizability is wavenumber-dependent, $\zeta=\zeta(k)$. This can occur more generally with any BS.

\subsection{Moving scatterer}

The transfer matrix associated with a scatterer moving at a velocity $v$ can be derived in three steps: (1) transform the electromagnetic field into a frame moving with the instantaneous velocity $v$ of the BS; (2) invoke then the transfer matrix of an immobile scatterer; (3) and finally transform back to the laboratory frame. Of course, the transfer matrix approach in this case applies if and only if the instantaneous velocity $v$ can be considered constant, that is, it varies slowly on the time scale needed for the field amplitudes to reach their steady-state. In this case, an $x_{\mathrm{BS}}$ and $v$ dependent transfer matrix can be defined. The above three steps are written formally as

$$
\hat{M}=\hat{L}(-v) M_{0} \hat{L}(v),
$$

where the Lorentz-boost transformation, mixing the magnetic and electric field vectors, can be taken in linear order in $v / c$, which leads to ${ }^{15}$

$$
\hat{L}(v)=\left[\begin{array}{cc}
\left(1+\frac{v}{c}\right) \hat{P}_{-v} & 0 \\
0 & \left(1-\frac{v}{c}\right) \hat{P}_{v}
\end{array}\right] .
$$

Obviously, $\hat{L}^{-1}(v)=\hat{L}(-v)$ to first order in $\frac{v}{c}$. Here we defined the Doppler-shift operator $\hat{P}_{v}: f(k) \mapsto$ $f\left(k+k \frac{v}{c}\right)$, which acts in the space of the wave vectors rather than in the space of amplitudes. The corresponding displacement in the wave vector space represents the Doppler effect: the moving BS couples counter-propagating waves differing in frequency by an amount of $2 \omega \frac{v}{c}$, i.e., $C(k)$, is combined with the amplitudes $A(k)$ and $B(k-$ $\left.2 k \frac{v}{c}\right)$. A similar statement holds for $D(k)$. The transfer matrix in the laboratory frame can thus be conceived as a 2-by-2 supermatrix acting also in the $k$-space.

Later we will use the expansion of the Doppler-shift operator to first order in $v / c$, which is

$$
\hat{P}_{v}=1+\frac{v}{c} k_{0} \frac{\partial}{\partial k},
$$

assuming that the populated modes in the whole system span a Doppler broadened, but narrow frequency range around the central pumping frequency $c k_{0}$. The forthcoming calculations involve then the differentiation with respect to the variable $k$. This effect appears in two ways. First, the polarizability function can be dependent 
on the wave vector $k$, which is generally called a dispersive medium. The transfer matrix of a moving scatterer with polarizability $\zeta(k)$ is

$$
M_{S}=\left[\begin{array}{cc}
1-i \zeta-i \frac{v}{c} k_{0} \frac{\partial \zeta}{\partial k} & -i \zeta\left[1-\frac{v}{c}\left(2-\frac{k_{0}}{\zeta} \frac{\partial \zeta}{\partial k}-2 k_{0} \frac{\partial}{\partial k}\right)\right] \\
i \zeta\left[1+\frac{v}{c}\left(2-\frac{k_{0}}{\zeta} \frac{\partial \zeta}{\partial k}-2 k_{0} \frac{\partial}{\partial k}\right)\right] & 1+i \zeta-i \frac{v}{c} k_{0} \frac{\partial \zeta}{\partial k}
\end{array}\right] .
$$

Second, the free propagation in a distance $d$ between two scatterers is described by the transfer matrix

$$
M_{\mathrm{p}}=\left[\begin{array}{cc}
e^{i k d} & 0 \\
0 & e^{-i k d}
\end{array}\right],
$$

These $k$-dependent exponential functions are responsible for the Doppler-shift in the phase which can yield significant effects in interferometers.

\subsection{The force acting on the scatterer}

The force acting on the medium in the direction $x$ derives from the surface integral of the $x x$ component of the Maxwell stress tensor $\boldsymbol{T}$ on the surface, $\mathcal{S}$, of a fictitious volume $V=S \delta l$ enclosing the medium, where $S$ is the mode area and $\delta l$ the infinitesimal length of the volume along the ' $x$ ' axis (see Fig. 1). Then, this force is given by

$$
\boldsymbol{F}=\oint_{\mathcal{S}} \boldsymbol{T}_{x x} n_{x} \mathrm{~d} \mathcal{S}=S\left[\boldsymbol{T}_{x x}\left(x \rightarrow 0^{+}\right)-\boldsymbol{T}_{x x}\left(x \rightarrow 0^{-}\right)\right],
$$

where the BS is at $x_{\mathrm{BS}}=0$, and $n_{x}=\operatorname{sgn}(x)$ is the normal to $\mathcal{S}$. From the definition of the Maxwell stress tensor, it follows that ${ }^{15}$

$$
\boldsymbol{T}_{x x}=-2 \epsilon_{0}\left[\left|\sum_{k} A(k) e^{-i k x-i \omega t}\right|^{2}+\left|\sum_{k} B(k) e^{i k x-i \omega t}\right|^{2}\right],
$$

for $x<0$. Assuming a narrow bandwidth of populated modes around the central wavenumbers $\pm k_{0}$, the force expression reduces to

$$
\boldsymbol{F}=\frac{\hbar \omega}{c}\left(|A|^{2}+|B|^{2}-|C|^{2}-|D|^{2}\right),
$$

where $A=\left[\hbar \omega /\left(2 S \epsilon_{0} c\right)\right]^{-1 / 2} \sum_{k} A(k)$ is the photo-current amplitude, and similarly for $B, C$ and $D$, their modulus square giving the number of photons crossing a unit surface per unit time. Although we considered first the electric field composed of independent modes, in the force expression only the sums of the mode amplitudes occur.

\subsection{Transfer matrix of a composite system of scatterers}

The main virtue of the transfer matrix approach is the possibility of cascading several consecutive scatterers. An arbitrary one-dimensional configuration of optical elements can be treated by simply multiplying 2-by-2 matrices. We will assume that only one of the scatterers is moving and we are interested in the force acting on this one. Then the appropriate notation is summarized in Fig. 2. The transfer matrix $\hat{M}(k)$ of the entire system is given by the product $\hat{M}(k)=M_{1}(k) \hat{M}_{\mathrm{S}}(k) M_{2}(k)$, where the transfer matrices $\hat{M}_{\mathrm{S}}(k)$ and $M_{1,2}(k)$ stand for the scatterer and for the general optical systems preceding and following the scatterer, respectively. We then calculate the inverse of the transfer matrix $M_{1}(k)$, such that

$$
\left(\begin{array}{l}
A_{1} \\
B_{1}
\end{array}\right)=\hat{M}\left(\begin{array}{c}
C_{\mathrm{r}} \\
D_{\mathrm{r}}
\end{array}\right) \text { and }\left(\begin{array}{l}
A \\
B
\end{array}\right)=M_{1}^{-1}\left(\begin{array}{l}
A_{1} \\
B_{1}
\end{array}\right)
$$

where we have omitted the $k$-dependence. We use the hat to indicate that the corresponding matrix contains the Doppler shift operator $\hat{P}_{v}$. The elements of these matrices, which we denote by

$$
M_{1}^{-1} \equiv\left[\theta_{i j}\right], i, j=1,2, \text { and } \hat{M} \equiv\left[\begin{array}{cc}
\hat{\gamma} & \hat{\alpha} \\
\hat{\delta} & \hat{\beta}
\end{array}\right]
$$


(a)

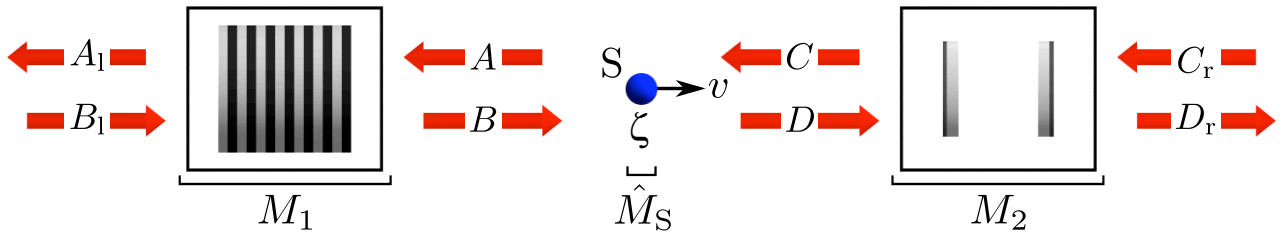

(b)

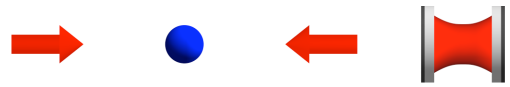

(c)

Figure 2. (a) General one-dimensional configuration of optical elements with one scatterer moving. The scatterer $S$ is between two sets of generic immobile optical elements (we show a Bragg reflector, on the left, and a Fabry-Pérot-type cavity, on the right, as an example). (b) External cavity cooling configuration, discussed in Section 4. (c) Mirror mediated cooling configuration, discussed inSection 3.

can all be obtained in a straightforward manner using only 2-by-2 matrix multiplication for an arbitrary number of scatterers, and hence can in principle be calculated analytically.

In order to make the mathematics more concise, we consider the case where we pump the system from only one direction. Setting $C_{\mathrm{r}}(k)=0$ in Eq. (14), we obtain

$$
A_{1}(k)=\hat{\alpha} \hat{\beta}^{-1} B_{1}(k) .
$$

Because of the presence of $\hat{\beta}^{-1}$, this relation between the back-reflected and the incoming fields contains the powers of the shift operator $\hat{P}_{v}$ to all orders. However, both $\hat{\alpha}$ and $\hat{\beta}$ can be expanded up to linear order in $v / c$,

$$
\hat{\alpha}=\alpha_{0}+\frac{v}{c}\left(\alpha_{1}^{(0)}+\alpha_{1}^{(1)} \frac{\partial}{\partial k}\right) \text { and } \hat{\beta}=\beta_{0}+\frac{v}{c}\left(\beta_{1}^{(0)}+\beta_{1}^{(1)} \frac{\partial}{\partial k}\right) .
$$

The auxiliary functions $\alpha_{0}, \alpha_{1}^{(0)}, \ldots$, are simply related to the matrix elements defined in Eq. (15) and to the scattering strength parameter, or 'polarizability', $\zeta$, following from the expressions of the previous subsection. Thus $\hat{\beta}$ can be inverted in closed form up to linear order in $v / c$,

$$
\hat{\beta}^{-1}=\frac{1}{\beta_{0}}-\frac{v}{c}\left(\frac{\beta_{1}^{(0)}}{\beta_{0}^{2}}+\frac{\beta_{1}^{(1)}}{\beta_{0}} \frac{\partial}{\partial k} \frac{1}{\beta_{0}}\right),
$$

which yields then the amplitudes $A(k)$. According to the force expression Eq. (13), the total field amplitudes $\mathcal{A}=\int A(k) \mathrm{d} k$ and $\mathcal{B}=\int B(k) \mathrm{d} k$ are needed,

$$
\begin{aligned}
& \mathcal{A}=\mathcal{A}_{0}+\frac{v}{c} \mathcal{A}_{1}=\left[\left(\theta_{11} \frac{\alpha_{0}}{\beta_{0}}+\theta_{12}\right)+\frac{v}{c}\left(\theta_{11} \frac{\alpha_{1}^{(0)} \beta_{0}-\alpha_{0} \beta_{1}^{(0)}}{\beta_{0}^{2}}-\frac{1}{\beta_{0}} \frac{\partial}{\partial k} \theta_{11} \frac{\alpha_{1}^{(1)} \beta_{0}-\alpha_{0} \beta_{1}^{(1)}}{\beta_{0}}\right)\right] B_{0}, \\
& \mathcal{B}=\mathcal{B}_{0}+\frac{v}{c} \mathcal{B}_{1}=\left(\theta_{21} \frac{\alpha_{0}}{\beta_{0}}+\theta_{22}+\frac{v}{c}\left\{\frac{\theta_{21}}{\beta_{0}^{2}}\left(\alpha_{1}^{(0)} \beta_{0}-\alpha_{0} \beta_{1}^{(0)}\right)-\frac{1}{\beta_{0}}\left[\partial_{k} \frac{\theta_{21}}{\beta_{0}}\left(\alpha_{1}^{(1)} \beta_{0}-\alpha_{0} \beta_{1}^{(1)}\right)\right]\right\}\right) B_{0}
\end{aligned}
$$

The amplitudes on the right side of the moving scatterer can be expressed, using the elements of $\hat{M}_{\mathrm{S}}$, as

$$
\begin{aligned}
\mathcal{C} & =(1-i \zeta) \mathcal{A}-i \zeta\left(1-2 \frac{v}{c}\right) \mathcal{B}, \\
\mathcal{D} & =i \zeta\left(1+2 \frac{v}{c}\right) \mathcal{A}+(1+i \zeta) \mathcal{B},
\end{aligned}
$$

where we have used the explicit form of $\hat{M}_{\mathrm{S}}$, and where we have defined $\mathcal{C}=\int C(k) \mathrm{d} k$ and $\mathcal{D}=\int D(k) \mathrm{d} k$. In Eqs. (20) we have also assumed that $\zeta$ is independent of $k$. Upon using these relations, we obtain an expression for the force acting on the scatterer, from which we can extract the friction force:

$$
\begin{aligned}
\boldsymbol{F}_{1}=-4 \hbar k_{0} \frac{v}{c}\left[|\zeta|^{2}\left(\left|\mathcal{A}_{0}\right|^{2}-\left|\mathcal{B}_{0}\right|^{2}\right)+\left(|\zeta|^{2}+\operatorname{Im}\{\zeta\}\right) \operatorname{Re}\left\{\mathcal{A}_{0} \mathcal{A}_{1}^{\star}\right\}-2 \operatorname{Im}\{\zeta\} \operatorname{Re}\left\{\mathcal{A}_{0} \mathcal{B}_{0}^{\star}\right\}\right. \\
\left.+\left(|\zeta|^{2}-\operatorname{Im}\{\zeta\}\right) \operatorname{Re}\left\{\mathcal{B}_{0} \mathcal{B}_{1}^{\star}\right\}+\operatorname{Im}\{\zeta\} \operatorname{Re}\left\{\mathcal{A}_{0} \mathcal{B}_{1}^{\star}\right\}+\operatorname{Re}\left\{\left(|\zeta|^{2}+i \operatorname{Re}\{\zeta\}\right) \mathcal{A}_{1} \mathcal{B}_{0}^{\star}\right\}\right] .
\end{aligned}
$$


All our assumptions - i.e., pumping at a single wavenumber, frequency independent polarizability $(\partial \zeta / \partial k=0)$, and $C_{\mathrm{r}}(k)=0$ - are simplifying assumptions and can be relaxed.

\section{MOBILE SCATTERER IN FRONT OF A PERFECT MIRROR}

Consider the system in Fig. 3 where the scatterer, or 'atom', has a polarizability $\zeta$ uniform over the frequency range of interest. Compared to the general scheme Fig. 2, there is nothing on the left side of the scatterer $\left(M_{1}\right.$

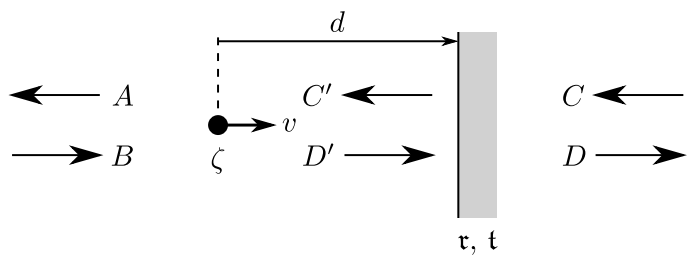

Figure 3. Schematic representation of the system composed of a fixed mirror and a moving scatterer in front of it. When the scatterer has a large polarizability, $|\zeta| \gg 1$, this system corresponds to a Fabry-Pérot resonator with one of its mirror moving. When $|\zeta| \ll 1$, the system is equivalent with that of a laser-driven atom interacting with its mirror image dipole.

is the unit matrix), and on the right side the total transfer matrix $M_{2}$ is composed of three elements. Letting $M_{\mathrm{a}}, M_{\mathrm{p}}$ and $M_{\mathrm{m}}$ be the transfer matrices for the atom, free propagation and mirror, respectively, we obtain the relation:

$$
\begin{gathered}
\left(\begin{array}{c}
A(k) \\
B(k)
\end{array}\right)=M_{\mathrm{a}} M_{\mathrm{p}} M_{\mathrm{m}}\left(\begin{array}{c}
C(k) \\
D(k)
\end{array}\right), \text { where } \\
M_{\mathrm{a}}=\left[\begin{array}{cc}
1+i \zeta & i \zeta\left(1-2 \frac{v}{c}\right) \hat{P}_{2 v} \\
-i \zeta\left(1+2 \frac{v}{c}\right) \hat{P}_{-2 v} & 1-i \zeta
\end{array}\right], \quad M_{\mathrm{p}}=\left[\begin{array}{cc}
e^{i k d} & 0 \\
0 & e^{-i k d}
\end{array}\right], \quad M_{\mathrm{m}}=\frac{1}{\mathfrak{t}}\left[\begin{array}{cc}
\mathfrak{t}^{2}-\mathfrak{r}^{2} & \mathfrak{r} \\
-\mathfrak{r} & 1
\end{array}\right] .
\end{gathered}
$$

The distance between the atom and the mirror is denoted by $d$. Note that the free propagation transfer matrix $M_{p}$ is non-uniform in the $k$-space, and therefore the Doppler-shift has an influence on the phase shift accumulated between two scattering events.

It is worth introducing the reference point at a distance $L=2 N \pi / k_{0}$ from the fixed mirror, where the integer $N$ is such that the moving atom's position $x$ is within a wavelength of this reference point. Then the atom-mirror distance can be replaced by $d=L-x$, and $k_{0} L$ drops from all the trigonometric functions. We need the sum of amplitudes, $\mathcal{A}=\int A(k) \mathrm{d} k / \mathcal{B}$, defined relative to the incoming amplitude $\mathcal{B}=\int B(k) d k$, which can be obtained in a closed form

$$
\begin{aligned}
& \mathcal{A}=\frac{1}{1-i \zeta}\left\{i \zeta+\mathfrak{r} \frac{e^{-2 i k_{0} x}}{1-i \zeta-\mathfrak{r} i \zeta e^{-2 i k_{0} x}}\right. \\
&\left.\quad-2 i \frac{v}{c} \zeta\left[1-\frac{\mathfrak{r}^{2} e^{-4 i k_{0} x}}{\left(1-i \zeta-\mathfrak{r} i \zeta e^{-2 i k_{0} x}\right)^{2}}-2 i k_{0}(L-x) \frac{\mathfrak{r}^{2}(1-i \zeta) e^{-4 i k_{0} x}}{\left(1-i \zeta-\mathfrak{r} i \zeta e^{-2 i k_{0} x}\right)^{3}}\right]\right\} .
\end{aligned}
$$

The main virtue of our approach is clearly seen, in that we can smoothly move from $\zeta=0$, which indicates the absence of the mobile scatterer, to $|\zeta| \rightarrow \infty$, which corresponds to a perfectly reflecting mirror, i.e., a moving boundary condition.

Using the definitions $\mathbb{A}=|\mathcal{A}|^{2}$ and $\mathbb{B}=|\mathcal{B}|^{2}$, the force acting on the moving scatterer in front of a mirror can be expressed as

$$
\boldsymbol{F}=-2 \hbar k_{0} \mathbb{B}\left(\left[|\zeta|^{2}\left(1+2 \frac{v}{c}\right)+\operatorname{Im}\{\zeta\}\right] \mathbb{A}+|\zeta|^{2}\left(1-2 \frac{v}{c}\right)-\operatorname{Im}\{\zeta\}+2 \operatorname{Re}\{i \zeta(1-i \zeta) \mathcal{A}\}\right),
$$

where $\mathcal{A}$ has to be substituted from Eq. (22). The coefficient of the term linear in velocity, the 'friction coefficient', is plotted in Fig. 4(a) as a function of the position $x$ in a half-wavelength range for various values of $\zeta$. When varying the coupling strength from $\zeta=0.01$ to $\zeta=1$, the friction coefficient transforms between two characteristic 

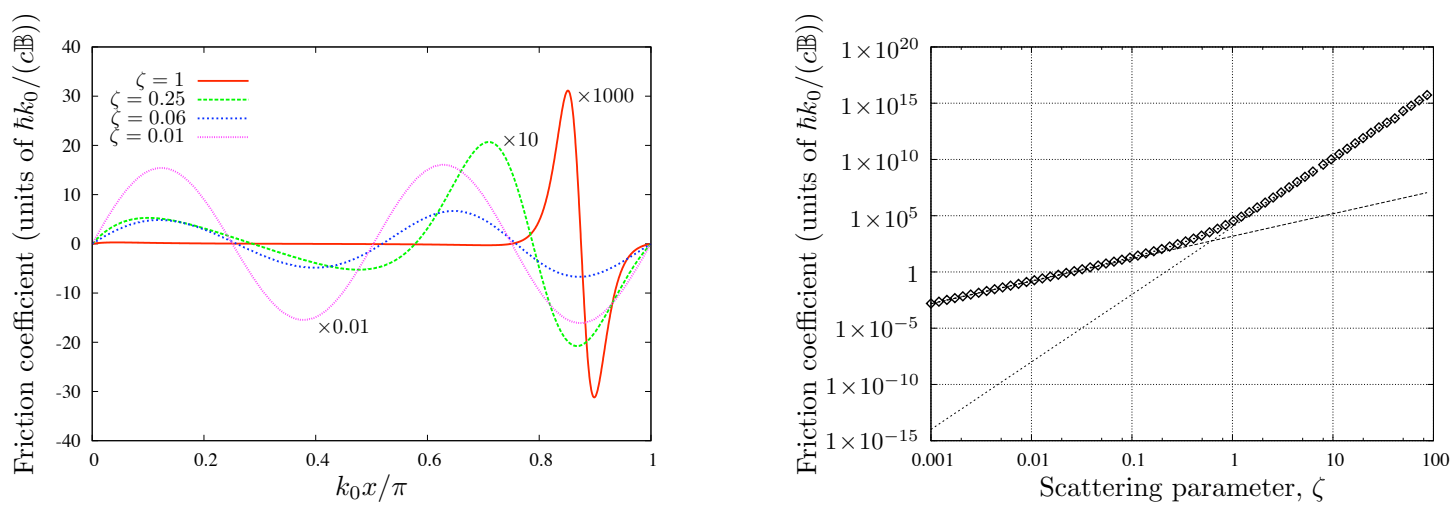

Figure 4. Left: The position dependence of the linear coefficient of the velocity-dependent force acting on the mobile scatterer in Fig. 3, for various scattering parameters $\zeta$, evaluated by using Eq. (22) and Eq. (23) with $k_{0} L=100$. The fixed mirror is assumed to be a perfect mirror. In order to fit all the curves into the same range, they are divided by the factors indicated in the figure. Right: The maximum friction force, taken in optimized position in a wavelength range, as a function of $\zeta$ (on a $\log$ - $\log$ scale) with $k_{0} L=100$. In the limit of small $\zeta$, the force scales as $\zeta^{2}$ (cf. Eq. (24); dashed line) whereas in the limit of large zeta it scales as $\zeta^{6}$ (cf. Eq. (26); dotted line).

regimes. For small coupling the linear velocity dependence tends to a simple sinusoidal function while, for large coupling, the friction exhibits a pronounced resonance in a narrow range. This resonance arises from the increased number of reflections between the mobile scatterer and the fixed mirror. It can be observed that the resonance shifts towards $k_{0} x=\pi$ on increasing $\zeta$. In the opposite limit of small $\zeta$, the maximum friction is obtained periodically at $\left(n-\frac{1}{4}\right) \pi / 2$ according to the sinusoidal function. The maximum friction force is plotted in Fig. 4(b), showing the two limiting cases of $\zeta^{2}$ behavior, in the limit of small $\zeta$, and $\zeta^{6}$ behavior, in the limit of large $\zeta$.

\subsection{Atom in front of a mirror: small $\zeta$ limit}

An atom pumped with a far off-resonance beam corresponds to a small and real $\zeta$, accordingly, we can truncate our expressions to second order in $\zeta$. We also assume, for simplicity, that the fixed mirror is perfect; i.e., $\mathfrak{r}=-1$ and $\mathfrak{t}=0$. Thus, the friction force simplifies to

$$
\boldsymbol{F}_{1}=-16 \hbar k_{0} \mathbb{B} \zeta^{2} \frac{v}{c}\left[\sin ^{2}\left(2 k_{0} x\right)-k_{0}(L-x) \sin \left(4 k_{0} x\right)\right],
$$

in full agreement with the result of a standard QED calculation. ${ }^{21}$ In the far field $(x \gg \lambda)$, the dominant friction term in the preceding expression is the last term, which renders the $\sin \left(4 k_{0} x\right)$ position dependence shown in Fig. 4(a) for $\zeta=0.01$.

\subsection{Fabry-Perot resonator with moving mirror: large $\zeta$ limit}

We will now consider the $|\zeta| \rightarrow \infty$ limit. We again assume that the fixed mirror of the resonator is perfect, with $\mathfrak{r}=-1$, and that the moving mirror has a real polarizability. The incoming field from the right can then be disregarded, i.e., $C=0$.

Let us first calculate the field in the resonator for $v=0$, which makes clear how to take the large $\zeta$ limit. This field is

$$
\mathcal{C}_{0}^{\prime}=(1-i \zeta) \mathcal{A}_{0}-i \zeta=-\frac{e^{-2 i \varphi}}{1-i \zeta+i \zeta e^{-2 i \varphi}}
$$

with $\varphi=k_{0} d$, which has a maximum at $\varphi_{0}$ obeying

$$
\tan \left(2 \varphi_{0}\right)=-\frac{1}{\zeta} .
$$

In the limit of $\zeta \rightarrow \infty$, the resonance is Lorentzian:

$$
\mathcal{C}_{0}^{\prime}=-\frac{e^{-2 i \varphi}}{2 i(1-i \zeta)\left[\left(\varphi-\varphi_{0}\right)-i \frac{1}{4 \zeta^{2}}\right]},
$$


with a width of $1 /\left(4 \zeta^{2}\right)$. This is the limit in which the field between the two mirrors can be considered a single-mode cavity field. After some algebra, the simplification of the force expression in Eq. (23) leads to the velocity-dependent terms

$$
\boldsymbol{F}_{1}=-\frac{1}{2} \frac{v}{c} \hbar k_{0}^{2} L \frac{\left(\varphi-\varphi_{0}\right)}{\zeta^{4}\left[\left(\frac{1}{4 \zeta^{2}}\right)^{2}+\left(\varphi-\varphi_{0}\right)^{2}\right]^{3}} \mathbb{B} .
$$

On substituting the usual cavity QED parameters, $\kappa=c /\left(4 L \zeta^{2}\right), \Delta_{C}=-c\left(\varphi-\varphi_{0}\right) / L, \eta^{2} /(2 \kappa)=\mathbb{B}$, and the optomechanical coupling constant $G=c^{2} k_{0}^{2} / L^{2}$, the friction force renders

$$
\boldsymbol{F}_{1}=-2 v \hbar G \operatorname{Re}\left\{a^{(0) \star} a^{(1)}\right\}=4 v \frac{\hbar \eta^{2} G^{2} \kappa \Delta_{C}}{\left[\Delta_{C}^{2}+\kappa^{2}\right]^{3}},
$$

which is the one derived from the usual radiation pressure Hamiltonian in the theory of optomechanics. ${ }^{16-18}$

\section{MOBILE SCATTERER IN FRONT OF A RESONATOR}

Consider the system in Fig. 5 where the scatterer is placed near an optical resonator, which we refer to as the 'external cavity cooling' configuration. We restrict ourselves to the special case of scatterers that can be characterized by a real polarizability $\zeta$; this is equivalent to assuming that no absorption takes place in the scatterer. The polarizability is further assumed to be uniform over the frequency range of interest. Note that in

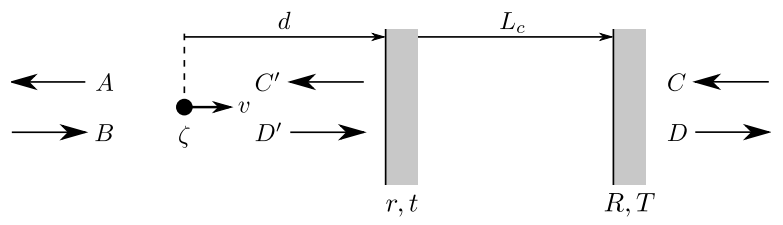

Figure 5. Schematic representation of the 'external cavity cooling' configuration. There are now two independent length scales, $L_{c}$ is the cavity length, and $d$ is the distance between the atom and the near mirror of the cavity.

this scheme with a near mirror of complex transmissivity $t$, the limits of small and large $|t|$ render the situation where the cavity is replaced respectively by the near mirror only or the far mirror only. That is, the results of the previous section can be reproduced in these limits. For intermediate $t$ compared with the transmissivity of the far mirror, $T$, the moving scatterer interacts with a field reflected back from the cavity and is subject to the interference created by the multiple reflections between the two mirrors. Here, we consider in particular an object having low reflectivity, around $50 \%$, which corresponds to a polarizability $\zeta=1$ and is representative of typical experimental conditions with micromirrors. ${ }^{6}$ This ensures that a high-finesse resonator cannot be formed between the object and the near mirror, thereby guaranteeing a parameter range where the cavity formed between the immobile mirrors dominates the interaction.

The spatial dependence of the friction force on the position of the scatterer exhibits two kinds of behavior on two distinct length scales. On a long scale, the gross spatial variation of the friction amplitude is linear in both $L_{c}$, the spacing between the two cavity mirrors, and $d$, the separation between the scatterer and the near mirror. This is simply because of the linear increase of the retardation time of the reflected field with this distance between the scatterer and the reflecting objects.

On a short scale corresponding to the optical wavelength $\lambda_{0}=2 \pi / k_{0}$, there is an essential modulation on top of the overall linear dependence of the friction force on both $d$ and $L_{c}$. First, external cavity cooling follows the same behavior as mirror mediated cooling (Section 3): there is a $\sin \left(4 k_{0} d\right)$ dependence. This implies that cooling occurs in regions of the size of $\lambda_{0} / 8$. In case of a micro-mechanical mirror, where the vibrational amplitude is naturally much less than the wavelength, the necessary confinement imposes no problem. Moreover, the simple sinusoidal form of the force means that the external cavity cooling scheme is exempt from the stability problems arising from the extreme sensitivity of the force on position that occurs when the moving mirror is inside the cavity. In the following, we will frequently refer to what we call the 'friction amplitude', which we define as the 


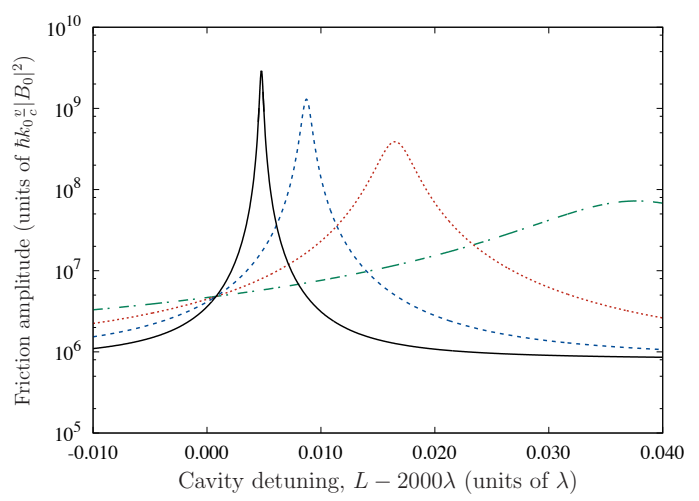

Figure 6. The amplitude of the friction force acting on the scatterer, for various near-mirror transmissivities, is shown as a function of the mirror separation in the cavity. The different curves represent different near-mirror transmissivities: $|t|=0.45$ (dashed-dotted curve), $|t|=0.20$ (dotted), $|t|=0.10$ (dashed), $|t|=0.05$ (solid). (Scatterer polarizability $\zeta=1$, scatterer-cavity separation $d \approx 400 \lambda_{0},|T|=0.01, \lambda_{0}=780 \mathrm{~nm}$.)

maximum of the friction force $-\boldsymbol{F}_{1}$ as one varies the position of the mobile scatterer over a wavelength-scale region.

As shown in Fig. 6, the fine tuning of the cavity length by varying $L_{c}$ on the wavelength scale produces a Lorentzian-like resonance of the friction amplitude. This resonance is intimately related to that in the intracavity field intensity. If we denote the complex reflectivities of the near and far mirror by $r$ and $R$, respectively, one can show that the field inside the cavity is maximized when the cavity length is

$$
L_{c}=m \frac{\lambda_{0}}{2}-\frac{1}{2 k_{0}} \arg (r R),
$$

with $m$ being an integer, which is a close approximation to the positions of the peaks in Fig. 6 . Moreover, the width of each of the resonance peaks in this figure is approximately equal to the width of the respective peak in the intra-cavity field intensity, which has a full-width at half-maximum given by $(1-|r R|) /\left(k_{0} \sqrt{|r R|}\right) \equiv \lambda_{0} /(2 \mathcal{F})$, where $\mathcal{F}$ is the cavity finesse. The resonant enhancement of the friction force amplitude can be attributed to a 'distance-folding' mechanism: the cavity effectively multiplies the retardation time by the number of round trips in the cavity. Note also that, in this situation, the optical path length is determined predominantly by the cavity length $L_{c}$, and is practically independent of $d$, even if $d$ and $L_{c}$ are of the same order of magnitude.

Since the total back-reflected field is composed of the interference of optical paths that have run different numbers of round trips within the external cavity, the force is not simply determined by the finesse, which measures the average number of round-trips. The complex dependence is investigated in Fig. 7, where the friction amplitude is plotted as a function of the near mirror transmissivity $|t|$ for a fixed far mirror transmissivity, $T=1 /(1-100 i)$. We note that this non-ideal reflectivity of the far mirror could equivalently arise from absorption, of ca. $0.01 \%$ with the given parameters, of the incident power by the mirror. The figure reveals that the friction force depends not only upon the retardation but also upon the cavity reflectivity, which drops near resonance in the well-known behavior of a Fabry-Pérot resonator. For each value of $|t|$, the cavity length $L_{c}$ has been adjusted to maximize the friction force, according to curves such as those in Fig. 6 . The calculated result follows the intra-cavity field (shown dashed) except where the cavity reflectivity drops near resonance (region (b)), and in the extremes of regions (a) and (c), where the geometry is dominated by the near $(|t| \rightarrow 0)$ or far $(|t| \rightarrow 1)$ mirrors, respectively. Fig. 7(b) shows the effect of the drop in reflectivity as the cavity is scanned through resonance for similar mirror reflectivities. When this causes a dip in the friction amplitude peak, the optimum values plotted in Fig. 7 occur to either side of the resonance, and the friction force in this region is effectively limited by this interference effect. We note that the friction amplitude is not maximized at the point of maximum intra-cavity field $(t=T)$ because more light is lost through the cavity for larger $|t|$.

In conclusion, with this example of 'external cavity cooling', we have proved that the light field in an interferometer is very sensitive to the motion of a scatterer, which leads to strongly enhanced friction force on the 

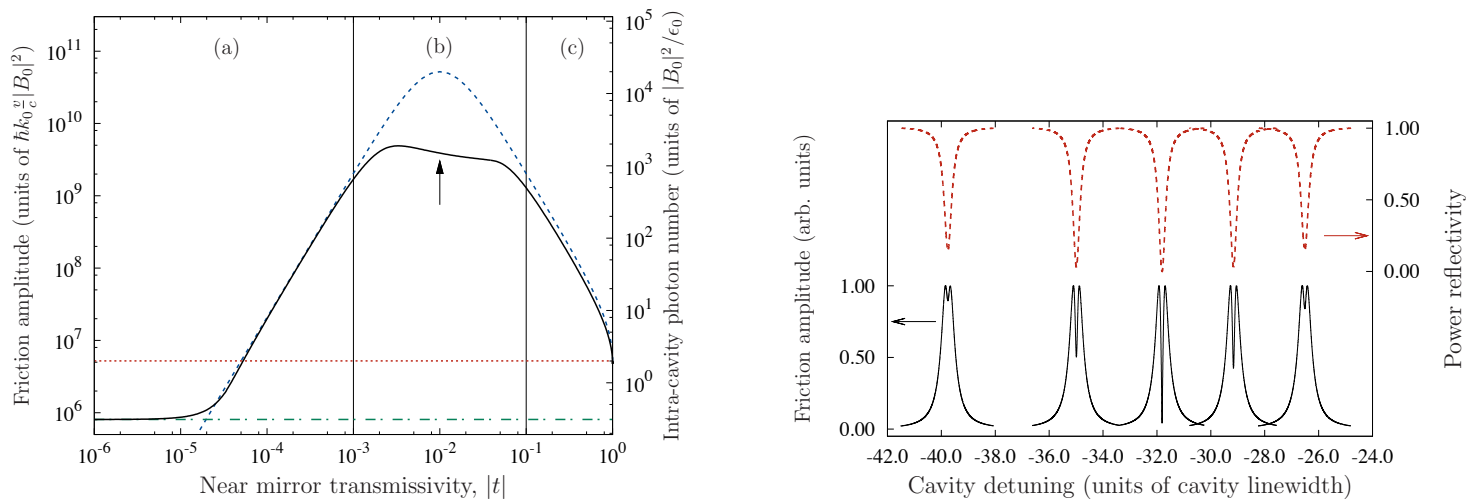

Figure 7. Left: Cavity enhancement of the friction force. The amplitude of the friction acting on a scatterer, with polarizability $\zeta=1.0$ (50\% reflectivity), interacting with a cavity tuned to achieve maximum friction, as we vary the transmissivity, $|t|$, of the near mirror. We compare the friction produced by such a cavity (black solid line) to mirror mediated cooling using the far mirror only (green dashed-dotted line) or the near mirror only (red dotted line), which correspond to the two limits $t=1$ and $t=0$, respectively. The vertical arrow indicates the point at which the two cavity mirrors have the same reflectivity. Also shown is the intra-cavity field (dashed blue line), acting as an aid to the eye. $|B|^{2}$ is the photon current of the incoming electric field. Right: In region (b) of the left panel, the friction coefficient amplitude (solid curves) is attenuated due to the attenuation in the field reflected from the cavity (dashed). $|T|=0.01$ in every plot; $|t|$ is, from left to right, $6.7 \times 10^{-3}, 8.3 \times 10^{-3}, 1.0 \times 10^{-2}, 1.2 \times 10^{-2}$, and $1.5 \times 10^{-2}$. (Scatterer-cavity separation $d \approx 400 \lambda_{0}$, cavity length $L \approx 2000 \lambda_{0},|T|=0.01, \lambda_{0}=780 \mathrm{~nm}$, finesse at peak friction $5.0 \times 10^{4}$.)

particle. This new cooling scheme may prove practical and efficient, and can be applied with large geometrical freedom on atoms as well as on micromirrors.

\section{ACKNOWLEDGMENTS}

This work was supported by the UK EPSRC (EP/E039839/1 and EP/E058949/1), by the CMMC collaboration within the EuroQUAM programme of the ESF, and by the National Office for Research and Technology (ERC_HU_09 OPTOMECH) of Hungary.

\section{REFERENCES}

[1] Marquardt, F. and Girvin, S. M., "Optomechanics," Physics 2, 40 (2009).

[2] Braginsky, V. B. and Manukin, A. B., [Measurement of Weak Forces in Physics Experiments], Univ of Chicago Pr (Tx), 1st ed. (1977).

[3] Schliesser, A., Arcizet, O., Rivière, R., Anetsberger, G., and Kippenberg, T. J., "Resolved-sideband cooling and position measurement of a micromechanical oscillator close to the Heisenberg uncertainty limit," Nat. Phys. 5, 509-514 (2009).

[4] Horak, P., Hechenblaikner, G., Gheri, K. M., Stecher, H., and Ritsch, H., "Cavity-induced atom cooling in the strong coupling regime," Phys. Rev. Lett. 79(25), 4974-4977 (1997).

[5] Domokos, P. and Ritsch, H., "Mechanical effects of light in optical resonators," J. Opt. Soc. Am. B 20, 1098-1130 (May 2003).

[6] Metzger, C. H. and Karrai, K., "Cavity cooling of a microlever," Nature 432, 1002-1005 (December 2004).

[7] Kleckner, D. and Bouwmeester, D., "Sub-kelvin optical cooling of a micromechanical resonator," $N a$ ture 444(7115), 75-78 (2006).

[8] Arcizet, O., Cohadon, P. F., Briant, T., Pinard, M., and Heidmann, A., "Radiation-pressure cooling and optomechanical instability of a micromirror," Nature 444(7115), 71-74 (2006).

[9] Gigan, S., Bohm, H. R., Paternostro, M., Blaser, F., Langer, G., Hertzberg, J. B., Schwab, K. C., Bauerle, D., Aspelmeyer, M., and Zeilinger, A., "Self-cooling of a micromirror by radiation pressure," Nature 444(7115), 67-70 (2006). 
[10] Law, C. K., "Interaction between a moving mirror and radiation pressure: A hamiltonian formulation," Phys. Rev. A 51(3), 2537-2541 (1995).

[11] Braginsky, V., "Low quantum noise tranquilizer for fabry-perot interferometer," Phys. Lett. A 293, 228-234 (February 2002).

[12] Deutsch, I. H., Spreeuw, R. J. C., Rolston, S. L., and Phillips, W. D., "Photonic band gaps in optical lattices," Physical Review A 52, 1394-1410 (Aug 1995).

[13] Asbóth, J. K., Ritsch, H., and Domokos, P., "Collective excitations and instability of an optical lattice due to unbalanced pumping," Phys. Rev. Lett. 98, 203008 (2007).

[14] Asbóth, J. K., Ritsch, H., and Domokos, P., "Optomechanical coupling in a one-dimensional optical lattice," Physical Review A (Atomic, Molecular, and Optical Physics) 77(6), 063424+ (2008).

[15] Xuereb, A., Domokos, P., Asbóth, J., Horak, P., and Freegarde, T., "Scattering theory of cooling and heating in optomechanical systems," Physical Review A 79,053810+ (May 2009).

[16] Wilson-Rae, I., Nooshi, N., Zwerger, W., and Kippenberg, T. J., "Theory of ground state cooling of a mechanical oscillator using dynamical backaction," Phys. Rev. Lett. 99(9), 093901 (2007).

[17] Marquardt, F., Chen, J. P., Clerk, A. A., and Girvin, S. M., "Quantum theory of cavity-assisted sideband cooling of mechanical motion," Phys. Rev. Lett. 99(9) (2007).

[18] Genes, C., Vitali, D., Tombesi, P., Gigan, S., and Aspelmeyer, M., "Ground-state cooling of a micromechanical oscillator: Comparing cold damping and cavity-assisted cooling schemes," Phys. Rev. A 77(3), 033804 (2008).

[19] Eschner, J., Raab, C., Schmidt-Kaler, F., and Blatt, R., "Light interference from single atoms and their mirror images," Nature 413(6855), 495-498 (2001).

[20] Bushev, P., Wilson, A., Eschner, J., Raab, C., Schmidt-Kaler, F., Becher, C., and Blatt, R., "Forces between a single atom and its distant mirror image," Phys. Rev. Lett. 92, 223602 (June 2004).

[21] Xuereb, A., Horak, P., and Freegarde, T., "Atom cooling using the dipole force of a single retroflected laser beam," Physical Review A 80, 013836+ (Jul 2009).

[22] Xuereb, A., Freegarde, T., Horak, P., and Domokos, P., "Optomechanical Cooling with Generalized Interferometers," Physical Review Letters 105, 013602+ (Jun 2010). 